\title{
703.
}

\section{ON THE ADDITION OF THE DOUBLE 9-FUNCTIONS.}

[From the Journal für die reine und angewandte Mathematik (Crelle), t. LxxxvIIr. (1879), pp. $74-81$.

I ASSUME in general

$$
\Theta=a-\theta \cdot b-\theta \cdot c-\theta \cdot d-\theta \cdot e-\theta \cdot f-\theta,
$$

and I consider the variables $x, y, z, w, p, q$, connected by the equations

$$
\left|\begin{array}{rrrrrr}
1, & 1, & 1, & 1, & 1, & 1 \\
x, & y, & z, & w, & p, & q \\
x^{2}, & y^{2}, & z^{2}, & w^{2}, & p^{2}, & q^{2} \\
x^{3}, & y^{3}, & z^{3}, & w^{3}, & p^{3}, & q^{3} \\
\sqrt{X}, & \sqrt{Y}, & \sqrt{Z}, & \sqrt{W}, & \sqrt{P}, & \sqrt{Q}
\end{array}\right|=0,
$$

equivalent to two independent equations, which in fact serve to determine $p$, $q$, or say the symmetrical functions $p+q$ and $p q$, in terms of $x, y, z, w$.

These equations, it is well known, constitute a particular integral of the differential equations

$$
\begin{aligned}
& \frac{d x}{\sqrt{X}}+\frac{d y}{\sqrt{Y}}+\frac{d z}{\sqrt{Z}}+\frac{d w}{\sqrt{W}}+\frac{d p}{\sqrt{P}}+\frac{d q}{\sqrt{Q}}=0, \\
& \frac{x d x}{\sqrt{X}}+\frac{y d y}{\sqrt{Y}}+\frac{z d z}{\sqrt{Z}}+\frac{w d w}{\sqrt{W}}+\frac{p d p}{\sqrt{P}}+\frac{q d q}{\sqrt{Q}}=0,
\end{aligned}
$$

or what is the same thing, regarding $p, q$ as arbitrary constants, they constitute the general integral of the differential equations

$$
\begin{aligned}
& \frac{d x}{\sqrt{X}}+\frac{d y}{\sqrt{Y}}+\frac{d z}{\sqrt{Z}}+\frac{d w}{\sqrt{W}}=0 \\
& \frac{x d x}{\sqrt{X}}+\frac{y d y}{\sqrt{Y}}+\frac{z d z}{\sqrt{Z}}+\frac{w d w}{\sqrt{W}}=0
\end{aligned}
$$


I attach the numbers $1,2,3,4,5,6$ to the variables $x, y, z, w, p, q$, respectively: and write

$$
A_{12}=\sqrt{a-x \cdot a-y} ; \quad A_{34}=\sqrt{a-z \cdot a-w} ; \quad A_{56}=\sqrt{a-p \cdot a-q} ;
$$

(six equations),

$A B_{12}=\frac{1}{x-y}\{\sqrt{a-x \cdot b-x \cdot f-x \cdot c-y \cdot d-y \cdot e-y}-\sqrt{a-y \cdot b-y \cdot f-y \cdot c-x \cdot d-x \cdot e-x}\} ;$ etc.

(ten equations),

where it is to be borne in mind that $A B$ is an abbreviation for $A B F . C D E$, and so in other cases, the letter $F$ belonging always to the expressed duad: there are thus in all the sixteen functions $A, B, C, D, E, F, A B, A C, A D, A E, B C, B D$, $B E, C D, C E, D E$, these being functions of $x$ and $y$, of $z$ and $w$, and of $p$ and $q$, according as the suffix is 12,34 , or 56 .

It is to be shown that the 16 functions $A_{56}, A B_{56}$ of $p$ and $q$ can be by means of the given equations expressed as proportional to rational and integral functions of the 16 functions $A_{12}, A B_{12}, A_{34}, A B_{34}$ of $x$ and $y$, and of $z$ and $w$ respectively: and it is clear that in so expressing them we have in effect the solution of the problem of the addition of the double 9 -functions.

I use when convenient the abbreviated notations

we have of course

$$
\begin{aligned}
a & -x=\mathrm{a}_{1}, \quad a-y=\mathrm{a}_{2}, \quad \text { etc., } \\
b & -x=\mathrm{b}_{1}, \quad \text { etc., } \\
\theta_{12} & =x-y, \quad \theta_{34}=z-w, \quad \theta_{56}=p-q ; \\
X & =\mathrm{a}_{1} \mathrm{~b}_{1} \mathrm{c}_{1} \mathrm{~d}_{1} \mathrm{e}_{1} \mathrm{f}_{1}, \\
A_{12} & =\sqrt{\mathrm{a}_{1} \mathrm{a}_{2}}, \\
A B_{12} & =\frac{1}{\theta_{12}}\left\{\sqrt{\mathrm{a}_{1} \mathrm{~b}_{1} \mathrm{t}_{1} \mathrm{c}_{2} \mathrm{~d}_{2} \mathrm{e}_{2}}-\sqrt{\mathrm{a}_{2} \mathrm{~b}_{2} \mathrm{f}_{2} \mathrm{c}_{1} \mathrm{~d}_{1} \mathrm{e}_{1}}\right\}, \text { etc. }
\end{aligned}
$$

Proceeding to the investigation, the equations between the-variables are obviously those obtained by the elimination of the arbitrary multipliers $\alpha, \beta, \gamma, \delta, \epsilon$ from the six equations obtained from

$$
\alpha \theta^{3}+\beta \theta^{2}+\gamma \theta+\delta=\epsilon \sqrt{\Theta}
$$

by writing therein for $\theta$ the values $x, y, z, w, p, q$ successively; we may consider the four equations

$$
\begin{aligned}
& \alpha x^{3}+\beta x^{2}+\gamma x+\delta=\epsilon \sqrt{\bar{X}}, \\
& \alpha y^{3}+\beta y^{2}+\gamma y+\delta=\epsilon \sqrt{\bar{Y}}, \\
& \alpha z^{3}+\beta z^{2}+\gamma z+\delta=\epsilon \sqrt{Z}, \\
& \alpha w^{3}+\beta w^{2}+\gamma w+\delta=\epsilon \sqrt{W},
\end{aligned}
$$


as serving to determine the ratios $\alpha: \beta: \gamma: \delta: \epsilon$ in terms of $x, y, z, w$; and we have then for the determination of $p, q$ the remaining two equations

$$
\begin{aligned}
& \alpha p^{3}+\beta p^{2}+\gamma p+\delta=\epsilon \sqrt{P}, \\
& \alpha q^{3}+\beta q^{2}+\gamma q+\delta=\epsilon \sqrt{Q},
\end{aligned}
$$

which two equations may be replaced by the identity

$$
\left(\alpha \theta^{3}+\beta \theta^{2}+\gamma \theta+\delta\right)^{2}-\epsilon^{2} \Theta=\alpha^{2}-\epsilon^{2} \cdot \theta-x \cdot \theta-y \cdot \theta-z \cdot \theta-w \cdot \theta-p \cdot \theta-q .
$$

Writing herein $\theta=$ any one of the values $a, b, c, d, e, f$, for instance $\theta=a$, and taking the square root of each side, we have

$$
\alpha a^{3}+\beta a^{2}+\gamma a+\delta=\sqrt{\alpha^{2}-\epsilon^{2}} \sqrt{a-x \cdot a-y} \sqrt{a-z \cdot a-w} \sqrt{a-p \cdot a-q},
$$

or as this may be written

$$
\alpha a^{3}+\beta a^{2}+\gamma a+\delta=\sqrt{\alpha^{2}-\epsilon^{2}} A_{12} \cdot A_{34} \cdot A_{56},
$$

which equation when reduced gives the proportional value of $A_{56}$.

For the reduction we require the value of $\alpha a^{3}+\beta a^{2}+\gamma a+\delta$ : calling this for the moment $\Omega$, we join to the four equations a fifth equation

$$
\alpha a^{3}+\beta a^{2}+\gamma a+\delta=\Omega
$$

Eliminating $\alpha, \beta, \gamma, \delta$, we find

or, what is the same thing,

$$
\begin{array}{lllll}
x^{3}, & x^{2}, & x, & 1, & \in \sqrt{X} \\
y^{3}, & y^{2}, & y, & 1, & \in \sqrt{Y} \\
z^{3}, & z^{2}, & z, & 1, & \in \sqrt{ } Z \\
w^{3}, & w^{2}, & w, & 1, & \in \sqrt{W} \\
a^{3}, & a^{2}, & a, & 1, & \Omega
\end{array} \mid=0,
$$

$$
\Omega\left|\begin{array}{cccc}
x^{3}, & x^{2}, & x, & 1 \\
y^{3}, & y^{2}, & y, & 1 \\
z^{3}, & z^{2}, & z, & 1 \\
w^{3}, & w^{2}, & w, & 1
\end{array}\right|+\epsilon\left|\begin{array}{ccccc}
\sqrt{X}, & x^{3}, & x^{2}, & x, & 1 \\
\sqrt{Y}, & y^{3}, & y^{2}, & y, & 1 \\
\sqrt{Z,} & z^{3}, & z^{2}, & z, & 1 \\
\sqrt{W}, & w^{3}, & w^{2}, & w, & 1 \\
& a^{3}, & a^{2}, & a, & 1
\end{array}\right|=0
$$

viz. this is

$\Omega \cdot x-y \cdot x-z \cdot x-w \cdot y-z \cdot y-w \cdot z-w=-\epsilon\{\sqrt{X} \cdot y-z \cdot y-w \cdot y-a \cdot z-w \cdot z-a \cdot w-a$

$$
\begin{aligned}
& +\sqrt{Y} \cdot z-w \cdot z-a \cdot z-x \cdot w-a \cdot w-x \cdot a-x \\
& +\sqrt{Z} \cdot w-a \cdot w-x \cdot w-y \cdot a-x \cdot a-y \cdot x-y \\
& +\sqrt{W} \cdot a-x \cdot a-y \cdot a-z \cdot x-y \cdot x-z \cdot y-z\}
\end{aligned}
$$

c. $\mathrm{x}$. 
or as it may be written

$\Omega \cdot x-z \cdot x-w \cdot y-z \cdot y-w=\frac{\epsilon \cdot a-z \cdot a-w}{x-y}\{y-z \cdot y-w \cdot a-y \cdot \sqrt{ } X-x-z \cdot x-w \cdot a-x \cdot \sqrt{ } Y\}$

$$
+\frac{\epsilon \cdot a-x \cdot a-y}{z-w}\{w-x \cdot w-y \cdot a-w \cdot \sqrt{Z}-z-x \cdot z-y \cdot a-z \cdot \sqrt{W}\},
$$

an equation for the determination of $\Omega$.

Consider first the expression which multiplies $\epsilon . a-z \cdot a-w$; this is

$$
=\frac{1}{\theta_{12}}\left\{y-z \cdot y-w \cdot a_{2} \sqrt{X}-x-z \cdot x-w \cdot a_{1} \sqrt{Y}\right\} ;
$$

we have

$$
B E_{12}=\frac{1}{\theta_{12}}\left\{\sqrt{\mathrm{b}_{1} \mathrm{e}_{1} \mathrm{f}_{1} \mathrm{a}_{2} \mathrm{c}_{2} \mathrm{~d}_{2}}-\sqrt{\mathrm{b}_{2} \mathrm{e}_{2} \mathrm{f}_{2} \mathrm{a}_{1} \mathrm{c}_{1} \mathrm{~d}_{1}}\right\}
$$

and multiplying this by

$$
A_{12} \cdot C_{12} \cdot D_{12},=\sqrt{\mathrm{a}_{1} \mathrm{c}_{1} \mathrm{~d}_{1} \mathrm{a}_{2} \mathrm{c}_{2} \mathrm{~d}_{2}}
$$

we derive

$$
B E_{12} . C_{12} . D_{12} . A_{12}=\frac{1}{\theta_{12}}\left\{c_{2} d_{2} a_{2} \sqrt{X}-c_{1} d_{1} a_{1} \sqrt{Y}\right\}
$$

and similarly two other equations; the system may be written

$$
\begin{aligned}
& \text { BE.C.D.A }=\frac{1}{\theta_{12}}\left\{\mathrm{c}_{2} \mathrm{~d}_{2} \mathrm{a}_{2} \sqrt{X}-\mathrm{c}_{1} \mathrm{~d}_{1} \mathrm{a}_{1} \sqrt{Y}\right\}, \\
& C E \cdot D \cdot B \cdot A="\left\{\mathrm{~d}_{2} \mathrm{~b}_{2}, "-\mathrm{d}_{1} \mathrm{~b}_{1}, "\right\}, \\
& D E \cdot B \cdot C \cdot A="\left\{\mathrm{~b}_{2} \mathrm{c}_{2}, "-\mathrm{b}_{1} \mathrm{c}_{1}, "\right\},
\end{aligned}
$$

the suffixes on the left-hand side being always 12 . The letters $b, c, d$ which enter cyclically into these equations are any three of the five letters other than $a$; the remaining two letters $e$ and $f$ enter symmetrically, for $B E$ is a mere abbreviation for the double triad $B E F . A C D$; and the like for $C E$, and $D E$.

Multiplying these equations by

$$
\frac{b-z \cdot b-w}{b-c \cdot b-d}, \quad \frac{c-z \cdot c-w}{c-d \cdot c-b}, \quad \frac{d-z \cdot d-w}{d-b \cdot d-c}
$$

respectively, and then adding, the right-hand side becomes

Writing

$$
=\frac{1}{\theta_{12}}\left\{y-z \cdot y-w \cdot a_{2} \sqrt{X}-x-z \cdot x-w \cdot a_{1} \sqrt{Y}\right\} .
$$

$$
\frac{b-z \cdot b-w}{b-c \cdot b-d}=\frac{-1}{c-d \cdot d-b \cdot b-c} \cdot c-d \cdot B_{34}{ }^{2}, \text { etc. }
$$


the left-hand side becomes

$$
=\frac{-A_{12}}{c-d \cdot d-b \cdot b-c}\left\{c-d \cdot B_{34}{ }^{2} \cdot B E_{12} \cdot C_{12} \cdot D_{12}+d-b \cdot C_{34}{ }^{2} \cdot C E_{12} \cdot D_{12} \cdot B_{12}+b-c \cdot D_{34}{ }^{2} \cdot D E_{12} \cdot B_{12} \cdot C_{12}\right\} \text {, }
$$

which for shortness may be written

$$
=\frac{-A_{12}}{c-d \cdot d-b \cdot b-c} \Sigma\left\{c-d \cdot B_{34}{ }^{2} \cdot B E_{12} \cdot C_{12} \cdot D_{12}\right\},
$$

the summation referring to the three terms obtained by the cyclical interchange of the letters $b, c, d$. The result thus is

$$
\begin{aligned}
& \frac{1}{\theta_{12}}\left\{y-z \cdot y-w \cdot \mathrm{a}_{2} \sqrt{X}-x-z \cdot x-w \cdot \mathrm{a}_{1} \sqrt{Y}\right\} \\
= & \frac{-A_{12}}{c-d \cdot d-b \cdot b-c} \Sigma\left\{c-d \cdot B_{34}{ }^{2} \cdot B E_{12} \cdot C_{12} \cdot D_{12}\right\} .
\end{aligned}
$$

Interchanging $x, y$ with $z, w$ respectively, we have of course to interchange the suffixes 1,2 and 3,4 ; we thus find

$$
\begin{aligned}
& \frac{1}{\theta_{34}}\left\{w-x \cdot w-y \cdot a_{4} \sqrt{Z}-z-x \cdot z-y \cdot a_{3} \sqrt{W}\right\} \\
= & \frac{-A_{34}}{c-d \cdot d-b \cdot b-c} \Sigma\left\{c-d \cdot B_{12}{ }^{2} \cdot B E_{34} \cdot C_{34} \cdot D_{34}\right\},
\end{aligned}
$$

and we hence find the value of $\Omega \cdot x-z \cdot x-w \cdot y-z \cdot y-w$. But $\Omega,=\alpha a^{3}+\beta a^{2}+\gamma a+\delta$, is $=\sqrt{\alpha^{2}-\epsilon^{2}} \cdot A_{12} \cdot A_{34} \cdot A_{56}$ : the resulting equation divides by $A_{12} \cdot A_{34}$ : throwing out this factor, we have

$$
\begin{gathered}
-\frac{\sqrt{\alpha^{2}-\epsilon^{2}}}{\epsilon}(x-z \cdot x-w \cdot y-z \cdot y-w)(c-d \cdot d-b \cdot b-c) A_{56} \\
=A_{34} \Sigma\left\{c-d \cdot B_{34}{ }^{2} \cdot B E_{12} \cdot C_{12} \cdot D_{12}\right\}+A_{12} \Sigma\left\{c-d \cdot B_{12}{ }^{2} \cdot B E_{34} \cdot C_{34} \cdot D_{34}\right\},
\end{gathered}
$$

where, as before, the summations refer to the three terms obtained by the cyelical interchange of the letters $b, c, d$; these being any three of the five letters other than $a$; and the remaining two letters $e, f$ enter into the formula symmetrically. The formula gives thus for $A_{56}$ ten values which are of course equal to each other.

Writing for $a$ each letter in succession, we obtain formulæ for each of the six single-letter functions $A_{56}$ of $p$ and $q$; and the factor

$$
-\frac{\sqrt{\alpha^{2}-\epsilon^{2}}}{\epsilon}(x-z \cdot x-w \cdot y-z \cdot y-w)
$$

is the same in all the formulæ.

We require further the expressions for the double-letter functions of $p$, $q$. Considering for example the function $D E_{56}$, which is

$$
=\frac{1}{\theta_{56}}\left\{\sqrt{d_{5} e_{5} f_{5} a_{6} b_{6} c_{6}}-\sqrt{d_{6} e_{6} f_{6} a_{5} b_{5} c_{5}}\right\}
$$


then multiplying by

we have

$$
A_{56} . B_{56} . C_{56},=\sqrt{\mathrm{a}_{5} \mathrm{~b}_{5} \mathrm{c}_{5} \mathrm{a}_{6} \mathrm{~b}_{6} \mathrm{c}_{6}}
$$

$$
\begin{gathered}
D E_{56} \cdot A_{56} \cdot B_{56} \cdot C_{56}=\frac{1}{\theta_{56}}\left\{\mathrm{a}_{6} \mathrm{~b}_{6} \mathrm{c}_{6} \sqrt{P}-\mathrm{a}_{5} \mathrm{~b}_{5} \mathrm{c}_{5} \sqrt{Q}\right\}, \\
=\frac{1}{p-q}\{a-q \cdot b-q \cdot c-q \cdot \sqrt{P}-a-p \cdot b-p \cdot c-p \cdot \sqrt{Q}\},
\end{gathered}
$$

or recollecting that $\epsilon \sqrt{P}, \epsilon \sqrt{Q}$ are $=\alpha p^{3}+\beta p^{2}+\gamma p+\delta$ and $\alpha q^{3}+\beta q^{2}+\gamma q+\delta$ respectively, this is

$$
\begin{gathered}
\epsilon \cdot D E_{56} \cdot A_{56} \cdot B_{56} \cdot C_{56} \\
=\frac{1}{p-q}\left\{a-q \cdot b-q \cdot c-q \cdot\left(\alpha p^{3}+\beta p^{2}+\gamma p+\delta\right)-a-p \cdot b-p \cdot c-p \cdot\left(\alpha q^{3}+\beta q^{2}+\gamma q+\delta\right)\right\} .
\end{gathered}
$$

Using the well-known identity

$$
\begin{aligned}
\alpha p^{3}+\beta p^{2}+\gamma p+\delta & =\alpha a^{3}+\beta a^{2}+\gamma a+\delta \cdot \frac{b-p \cdot c-p \cdot d-p}{b-a \cdot c-a \cdot d-a} \\
& +\alpha b^{3}+\beta b^{2}+\gamma b+\delta \cdot \frac{c-p \cdot d-p \cdot a-p}{c-b \cdot d-b \cdot a-b} \\
& +\alpha c^{3}+\beta c^{2}+\gamma c+\delta \cdot \frac{d-p \cdot a-p \cdot b-p}{d-c \cdot a-c \cdot b-c} \\
& +\alpha d^{3}+\beta d^{2}+\gamma d+\delta \cdot \frac{a-p \cdot b-p \cdot c-p}{a-d \cdot b-d \cdot c-d}
\end{aligned}
$$

and the like expression for $\alpha q^{3}+\beta q^{2}+\gamma q+\delta$, there will be on the right-hand side terms involving

$$
\alpha a^{3}+\beta a^{2}+\gamma a+\delta, \quad \alpha b^{3}+\beta b^{2}+\gamma b+\delta, \quad \alpha c^{3}+\beta c^{2}+\gamma c+\delta:
$$

but the term in $\alpha d^{3}+\beta d^{2}+\gamma d+\delta$ will disappear of itself.

The term in $\alpha a^{3}+\beta a^{2}+\gamma a+\delta$ is

$$
\frac{1}{p-q} \frac{\alpha a^{3}+\beta a^{2}+\gamma a+\delta}{b-a \cdot c-a \cdot d-a} \cdot b-q \cdot c-q \cdot b-p \cdot c-p \cdot(a-q \cdot d-p-a-p \cdot d-q)
$$

where the expression in () is $=d-a \cdot p-q$ : hence the term is

which is

$$
=\frac{a a^{3}+\beta a^{2}+\gamma a+\delta}{b-a \cdot c-a} \cdot b-q \cdot c-q \cdot b-p \cdot c-p,
$$

$$
=\frac{\alpha a^{3}+\beta a^{2}+\gamma a+\delta}{b-a \cdot c-a} B_{56}{ }^{2} \cdot C_{56}^{2} .
$$

Forming the two other like terms, the equation is

$$
\begin{aligned}
\epsilon \cdot D E_{56} \cdot A_{56} \cdot B_{56} \cdot C_{56}= & \frac{\alpha a^{3}+\beta a^{2}+\gamma a+\delta}{b-a \cdot c-a} B_{56}{ }^{2} \cdot C_{56}{ }^{2} \\
& +\frac{\alpha b^{3}+\beta b^{2}+\gamma b+\delta}{c-b \cdot a-b} C_{56}{ }^{2} \cdot A_{56}{ }^{2} \\
& +\frac{\alpha c^{3}+\beta c^{2}+\gamma c+\delta}{a-c \cdot b-c} A_{56}{ }^{2} \cdot B_{56}{ }^{2}
\end{aligned}
$$


But the expressions

are

$$
\alpha a^{3}+\beta a^{2}+\gamma a+\delta, \quad \alpha b^{3}+\beta b^{2}+\gamma b+\delta, \quad a c^{3}+\beta c^{2}+\gamma c+\delta,
$$

$$
=\sqrt{\alpha^{2}-\epsilon^{2}} A_{12} \cdot A_{34} \cdot A_{56}, \quad \sqrt{\alpha^{2}-\epsilon^{2}} B_{12}, B_{34} \cdot B_{56}, \quad \sqrt{\alpha^{2}-\epsilon^{2}} C_{12} \cdot C_{34} \cdot C_{56},
$$

respectively: the whole equation thus divides by $A_{56} . B_{56} . C_{56}$; throwing out this factor, and then multiplying each side by $-\frac{\sqrt{\alpha^{2}-\epsilon^{2}}}{\epsilon}$, we find

$$
\begin{aligned}
& -\frac{\sqrt{\alpha^{2}-\epsilon^{2}}}{\epsilon} D E_{56}=\frac{1}{b-c \cdot c-a \cdot a-b}\left(-\frac{\sqrt{\alpha^{2}-\epsilon^{2}}}{\epsilon}\right)^{2}\left\{b-c \cdot A_{12} \cdot A_{34} \cdot B_{56} \cdot C_{56}\right. \\
& +c-a \cdot B_{12} \cdot B_{34} \cdot C_{56} \cdot A_{56} \\
& \left.+a-b \cdot C_{12} \cdot C_{34} \cdot A_{56} \cdot B_{56}\right\}
\end{aligned}
$$

in which formula if we imagine

$$
-\frac{\sqrt{\alpha^{2}-\epsilon^{2}}}{\epsilon} A_{56}, \quad-\frac{\sqrt{\alpha^{2}-\epsilon^{2}}}{\epsilon} B_{56}, \quad-\frac{\sqrt{\alpha^{2}-\epsilon^{2}}}{\epsilon} C_{56}
$$

each replaced by its value in terms of the $x y$ - and $z w$-functions, we have an equation of the form

$$
-\frac{\sqrt{\alpha^{2}-\epsilon^{2}}}{\epsilon}(x-z \cdot x-w \cdot y-z \cdot y-w) D E_{56}=\frac{1}{x-z \cdot x-w \cdot y-z \cdot y-w} M,
$$

where $M$ is a given rational and integral function of the 16 and 16 functions $A_{12}, A B_{12}$ and $A_{34}, A B_{34}$ of $x$ and $y$ and of $z$ and $w$ respectively. The factor

$$
-\frac{\sqrt{\alpha^{2}-\epsilon^{2}}}{\epsilon}(x-z \cdot x-w \cdot y-z \cdot y-w)
$$

is retained on the left-hand side as being the same factor which enters into the equations for $A_{56}$, etc.: but on the right-hand side $x-z \cdot x-w \cdot y-z \cdot y-w$ should be expressed in terms of the $x y$ - and $z w$-functions. This can be done by means of the identity

$$
x-z \cdot x-w \cdot y-z \cdot y-w=\Sigma \frac{\left|\begin{array}{l}
1, x+y, x y \\
1, z+w, z w \\
1, a+b, a b
\end{array}\right|\left|\begin{array}{l}
1, x+y, x y \\
1, z+w, z w \\
1, a+c, a c
\end{array}\right|}{a-b \cdot a-c},
$$

where the summation refers to the three terms obtained by the cyclical interchange of the letters $a, b, c$. The first determinant, multiplied by $a-b$, is in fact

$$
=\left|\begin{array}{cc}
a-z \cdot a-w, & a-x \cdot a-y \\
b-z \cdot b-w, & b-x \cdot b-y
\end{array}\right|,
$$

and the second determinant, multiplied by $a-c$, is

$$
=\left|\begin{array}{cc}
a-z \cdot a-w, & a-x \cdot a-y \\
c-z \cdot c-w, & c-x \cdot c-y
\end{array}\right|
$$


so that the formula may also be written

$$
x-z \cdot x-w \cdot y-z \cdot y-w=\Sigma \frac{\left|\begin{array}{cc}
a-z \cdot a-w, & a-x \cdot a-y \\
b-z \cdot b-w, & b-x \cdot b-y
\end{array}\right| \cdot\left|\begin{array}{cc}
a-z \cdot a-w, & a-x \cdot a-y \\
c-z \cdot c-w, & c-x \cdot c-y
\end{array}\right|}{(a-b)^{2}(a-c)^{2}}
$$

or, what is the same thing, it is

$$
x-z \cdot x-w \cdot y-z \cdot y-w=\Sigma \frac{\left(A_{34}{ }^{2} B_{12}{ }^{2}-A_{12}{ }^{2} B_{34}{ }^{2}\right)\left(A_{34}{ }^{2} C_{12}{ }^{2}-A_{12}{ }^{2} C_{34}{ }^{2}\right)}{(a-b)^{2}(a-c)^{2}},
$$

which is the required expression for $x-z \cdot x-w \cdot y-z \cdot y-w$; the letters $a, b, c$, which enter into the formula, are any three of the six letters.

As regards the verification of the identity, observe that it may be written

$$
x-z \cdot x-w \cdot y-z \cdot y-w=\Sigma \frac{\{L+M(a+b)+N a b\}\{L+M(a+c)+N a c\}}{a-b \cdot a-c},
$$

where $L, M, N$ are

$$
=(x+y) z w-(z+w) x y, \quad x y-z w, \text { and } z+w-x-y:
$$

this is readily reduced to

$$
x-z \cdot x-w \cdot y-z \cdot y-w=M^{2}-N L,
$$

which can be at once verified.

Cambridge, 12th March, 1879.

I take the opportunity of remarking that, in the double-letter formulæ, the sign of the second term is, not as I have in general written it - , but is + ,

$$
A B=\frac{1}{x-y}\left\{\sqrt{\mathrm{abfc}_{1} \mathrm{~d}_{1} \mathrm{e}_{1}}+\sqrt{\mathrm{a}_{1} \mathrm{~b}_{1} \mathrm{f}_{1} \mathrm{cde}}\right\}, \text { etc. }
$$

In fact, introducing a factor $\omega$ which is a function of $x$ and $y$, the odd and even T-functions are $=\omega{\sqrt{a a_{1}}}_{1}$, etc., and

$$
\frac{\omega}{x-y}\left\{\sqrt{\mathrm{abfc}_{1} \mathrm{~d}_{1} \mathrm{e}_{1}}+\sqrt{\mathrm{a}_{1} \mathrm{~b}_{1} \mathrm{f}_{1} \mathrm{cde}}\right\}, \text { etc., }
$$

respectively; $\omega$ is a function which on the interchange of $x, y$ changes only its sign; and this being so, then when $x$ and $y$ are interchanged, each single-letter function changes its sign, and each double-letter function remains unaltered.

Cambridge, 29th July, 1879. 